\title{
Segmentação automática de lesões no cérebro em imagens de ressonância magnética usando superpixel, PSO e a rede geradora adversária com classificador auxiliar
}

\author{
Carolina L. S. Cipriano ${ }^{1}$, Giovanni L. F. da Silva ${ }^{1}$, Jonnison L. Ferreira ${ }^{1}$, \\ Aristófanes C. Silva ${ }^{1}$, Anselmo Cardoso de Paiva ${ }^{1}$ \\ ${ }^{1}$ Núcleo de Computação Aplicada - Universidade Federal do Maranhão (UFMA) \\ 65065-545 - São Luís - MA - Brasil \\ \{carol,giovannilucca, jonnison, ari, paiva\}@nca.ufma.br
}

\begin{abstract}
Gliomas are one of the most severe brain tumors. However, manual targeting is a difficult and time-consuming task. Therefore, this work proposes an automatic method for the segmentation of sub-regions of lesions in the brain in 3D MR images based on superpixels, PSO algorithm and the auxiliary generator network with auxiliary classifier. The proposed method obtained results for necrosis, edema, solid nucleus and nucleus, an accuracy of 67.71 $\%, 94.57 \%, 18.44 \%, 89.35 \%$ in the classification stage and coefficient dice of $60.35 \%, 44.22 \%, 16.45 \%, 31.23 \%$ in the segmentation stage for the respective subregions. The results demonstrate the difficulty in the classification and segmentation of the tumor sub-regions.
\end{abstract}

Resumo. Gliomas são um dos tumores cerebrais mais severos. No entanto, a segmentação manual é uma tarefa difícil e demorada. Portanto, esse trabalho propõe um método automático para a segmentação de sub-regiões de lesões no cérebro em imagens de RM 3D baseado em superpixels, algoritmo PSO e a rede geradora adversária com classificador auxiliar. O método proposto obteve como resultados para as sub-regióes necrose, edema, núcleo sólido e núcleo, uma acurácia de 67,71\%, 94,57\%, 18,44\%, 89,35\% na etapa de classificação e coeficiente dice de 60,35\%, 44,22\%, 16,45\%,31,23\% na etapa de segmentação para as respectivas sub-regiões. Os resultados demonstram a dificuldade na classificação e segmentação das sub-regiões tumorais.

\section{Introdução}

O cérebro e a medula espinhal formam o Sistema Nervoso Central (SNC). Os tumores do SNC devem-se ao crescimento de células anormais nos tecidos dessas localizações. O câncer do SNC representa de 1,4 a 1,8\% de todos os tumores malignos no mundo [Stewart et al. 2014]. Cerca de $88 \%$ dos tumores de SNC são no cérebro. Para o Brasil, estimam-se 5.810 novos casos de câncer do SNC em homens e 5.510 em mulheres para cada ano do biênio 2018-2019 [INCA 2019]. As taxas de sobrevida dos tumores cerebrais variam amplamente, dependendo do tipo de tumor, sua localização e estadiamento [Siegel et al. 2016].

Gliomas são tipos de tumores cerebrais que se originam das células gliais [Omuro and DeAngelis 2013]. Eles são o principal tipo de tumores cerebrais, em torno 
de 40 a 60\% das ocorrências [Goodenberger and Jenkins 2012]. Embora os gliomas sejam os tumores cerebrais mais comuns, eles podem ser menos agressivos, isto é, baixo grau em um paciente com expectativa de vida de vários anos ou mais agressivos, isto é, alto grau em um paciente com expectativa de vida de no máximo 2 anos [Havaei et al. 2017].

O diagnóstico precoce de gliomas desempenha um papel importante na melhoria das possibilidades do tratamento [Stewart et al. 2014]. Os exames por imagens como Tomografia Computadorizada (TC), Tomografia por Emissão de Positrões (PET) e Ressonância Magnética (RM) são usados para fornecerem informações valiosas sobre a forma, tamanho, localização e o metabolismo dos tumores cerebrais que auxiliam no diagnóstico. Embora essas modalidades sejam usadas em combinação para fornecerem mais informações sobre os tumores cerebrais, devido ao seu bom contraste dos tecidos moles e à ampla disponibilidade, a RM é considerada a técnica padrão [Işıı et al. 2016].

Diante disso, este trabalho propõe um método automático para a segmentação de lesões no cérebro em imagens de RM 3D baseado em superpixels, algoritmo de otimização de enxame de partículas, do inglês Particle Swarm Optimization (PSO), e a rede geradora adversária com classificador auxiliar, do inglês Auxiliary Classifier Generative Adversarial Network (ACGAN). Como contribuição desse trabalho, ressaltase o uso do algoritmo do PSO para otimização das técnicas de superpixels e seus respectivos parâmetros para uma melhor segmentação.

Este artigo está organizado da seguinte forma: a Seção 2 apresenta os trabalhos relacionados. A Seção 3 apresenta a metodologia proposta para a segmentação automática de lesões no cérebro. A Seção 4 apresenta e discute os resultados. Finalmente, a Seção 5 apresenta as considerações finais deste estudo.

\section{Trabalhos Relacionados}

Na literatura existem trabalhos relacionados a segmentação de lesões em imagens de RM, no qual estão divididos em duas principais abordagens sobre as regiões de interesse. A primeira abordagem trabalha com todas as sub-regiões da lesão (necrose, edema, núcleo sólido e núcleo). A segunda abordagem trabalha com 3 grandes regiões: completa (necrose, edema, núcleo sólido e núcleo), central (necrose, núcleo sólido e núcleo) e ativa (núcleo).

$\mathrm{Na}$ abordagem por sub-regiões, Alex et al. (2017) apresentou uma técnica semi-supervisionada para a detecção de lesões no cérebro usando as redes geradoras adversárias, do inglês Generative Adversarial Networks (GAN), extraindo patches dos quatro modos de RM da base de dados BraTS. A técnica proposta alcança um dice do tumor de $69 \%$. O coeficiente dice mede a similaridade da segmentação gerada pela rede e a marcação do especialista a nivel de pixel. Esse coeficiente é comumente utilizado para avaliar técnicas de segmentação [Dice 1945]. Além de uma sensibilidade de $91 \%$, especificidade de $59 \%$ e para várias sub-regiões média de $35 \%$ de acurácia, enquanto que a média de não lesão foi de $60 \%$.

O trabalho proposto por Lefkovits et al. (2016) utilizou um modelo discriminativo para detecção de tumores a partir de imagens de RM. A parte principal do modelo é construída em torno do classificador de floresta aleatória, do inglês Random Forest (RF). A sub-região necrose obteve uma acurácia média de $0 \%$, a sub-região edema obteve uma 
acurácia média de 36,99\%, a sub-região núcleo sólido obteve uma acurácia média de $1,96 \%$ e a sub-região núcleo obteve uma acurácia média de $0,86 \%$.

$\mathrm{Na}$ abordagem por grandes regiões, Pereira et al. (2016) propuseram um método de segmentação automática baseado em redes neurais convolucionais, do inglês Convolutional Neural Networks (CNN) e extração de patches obtendo dice de $88 \%, 83 \%$, $77 \%$ para as regiões completa, central e ativa, respectivamente.

Os métodos acima mencionados apresentam resultados razoáveis na segmentação de lesões cerebrais. No entanto, alguns trabalhos utilizam patches com sobreposição para extração do tecido da lesão e uma etapa de extração de características explícita. Esse procedimento apresenta dois problemas: 1) não garante a borda fidedigna da lesão e 2) alto tempo de processamento para extração dos patches e suas características. No trabalho proposto, nós utilizamos a técnica de superpixel otimizada pelo algoritmo de PSO para uma melhor delimitação do tecido de cada sub-região das lesões e a ACGAN para a classificação dos superpixels.

\section{Metodologia}

A metodologia proposta é dividida em quatro etapas, como ilustrado na Figura 1. Em resumo, a primeira etapa detalha os materiais usados como as imagens de RM. A segunda etapa consiste na aplicação das técnicas de melhoramento de imagens. Após esta etapa, a segmentação é realizada através da técnica de superpixels, o algoritmo de otimização de enxame de partículas e a rede geradora adversária com classificador auxiliar. Finalmente, os resultados são avaliados.
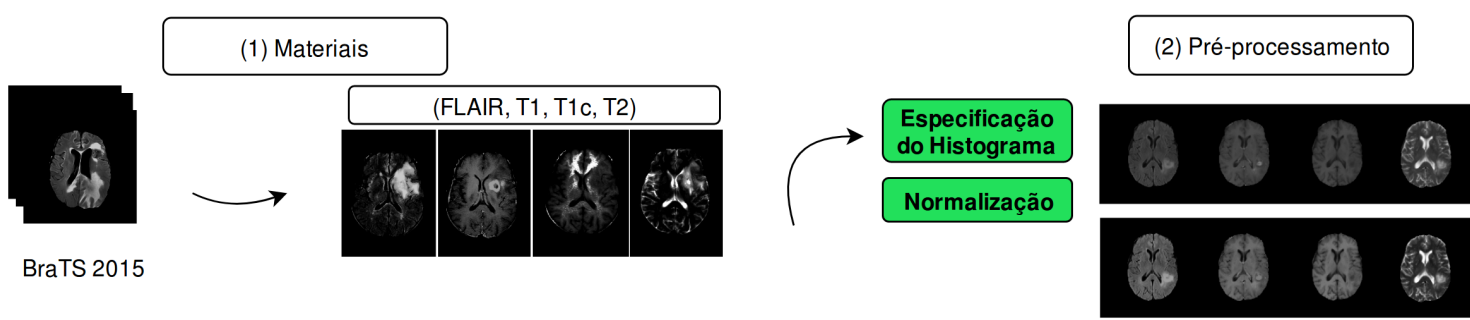

(3) Segmentação

(4) Validação

FLAIR, T1, T1c, T2
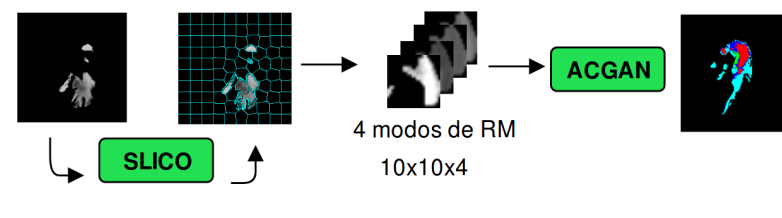

Acurácia

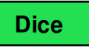

Figura 1. Metodologia proposta.

\subsection{Materiais}

A base de dados usada no trabalho é a Multimodal Brain Tumor Image Segmentation Benchmark (BrasTS) [Menze et al. 2015] composta por 220 pacientes contendo gliomas de alto grau. A marcação dos tumores foi realizada de forma manual por diversos especialistas e todos os pacientes compartilham de quatro modos de ressonância: T1, T1c, T2 e FLAIR [Morris and Slesnick 2018]. 
Além da marcação dos tumores cerebrais, a base disponibiliza a classificação da lesão em quatro sub-regiões: necrose, edema, núcleo sólido e núcleo. A Figura 2 (A) mostra as quatros sub-regiões com cores ilustrativas. Figura 2 (B) ilustra todo o tumor visto no modo FLAIR, Figura 2 (C) apresenta o núcleo sólido do tumor visível em T2, e por fim, Figura 2 (D) demonstra as estruturas tumorais visíveis em T1c em torno dos componentes necróticos do núcleo.
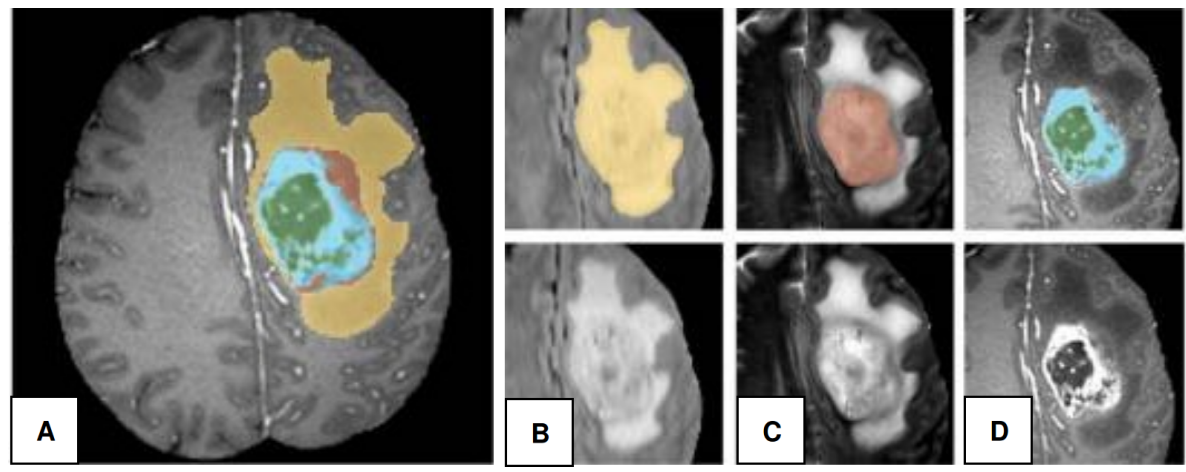

Figura 2. Lesão cerebral e suas sub-regiões. Edema (amarelo), núcleo sólido (vermelho), necrose (verde) e núcleo (azul) [Menze et al. 2015].

\subsection{Pré-processamento}

Antes da etapa de segmentação, duas técnicas de pré-processamento foram realizadas para normalizar os valores de intensidade das imagens de RM nas quatro modalidades. Como as imagens foram obtidas de diferentes equipamentos, os tecidos do cérebro podem apresentar intensidades muito diferentes em diferentes exames. Portanto, para reduzir esse tipo de problema inter-exame, a especificação do histograma é comumente aplicada, usando uma única imagem de RM de um paciente do banco de dados como referência para todos os outros. Então, o segundo passo consiste na normalização das intensidades intra-exame, com o objetivo de tornar a média próxima de zero e o desvio padrão menor [Pereira et al. 2016]. Para isso, é feita a divisão de todos os valores de pixel da fatia por sua média e o resultado é dividido pelo desvio padrão da fatia. A Figura 3 ilustra o resultado da etapa de pré-processamento.

\subsection{Segmentação}

A etapa de segmentação de lesões no cérebro é dividida em duas partes. A primeira parte consiste na aplicação da técnica de superpixels baseada no algoritmo de otimização de enxame de partículas. Depois disso, cada superpixel extraído é apresentado a uma rede geradora adversária com classificador auxiliar para a classificação das quatro sub-regiões do tumor cerebral.

\subsubsection{Extração dos superpixels baseado no algoritmo PSO}

A técnica de superpixel reduz significativamente o custo de memória, enquanto potencialmente aumenta a precisão de detecção, uma vez que geram regiões que correspondem a grupos pequenos e quase uniformes da imagem [Achanta et al. 2012]. Neste trabalho, as técnicas de superpixels analisadas foram o agrupamento iterativo linear 

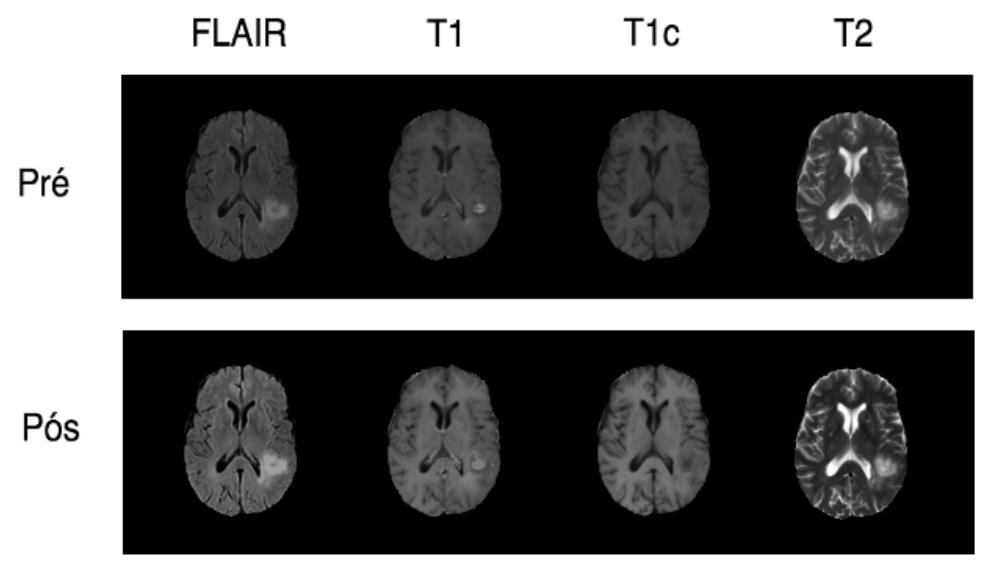

Figura 3. Aplicação da etapa do pre-processamento nas imagens de RM.

simples, do inglês Simple Linear Iterative Clustering (SLIC), e a sua variação SLIC otimizada (SLICO) [Diniz et al. 2018]. A Figura 4 apresenta a aplicação do SLIC na imagem de RM.
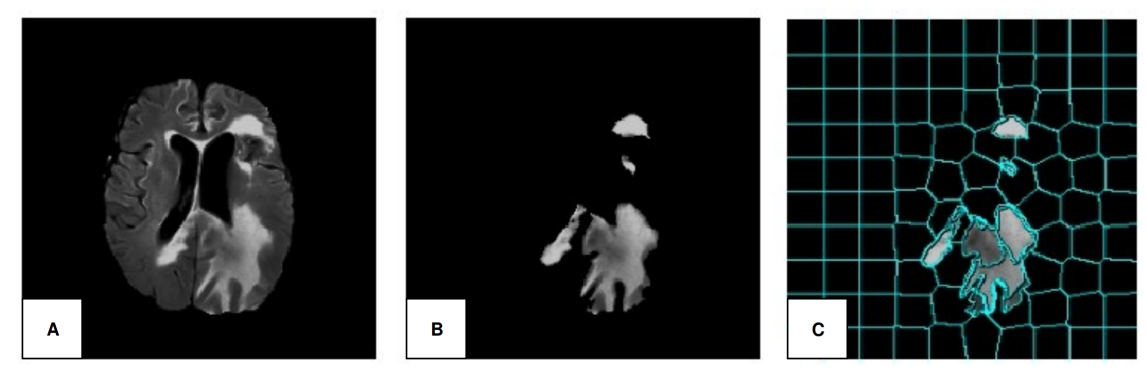

\section{Figura 4. (A) Imagem do cérebro no modo FLAIR, (B) lesão marcada pelo especialista e (C) SLIC aplicado a lesão.}

As duas técnicas de superpixels citadas (SLIC e SLICO) possuem dois parâmetros a serem inicializados. O primeiro, trata-se do tamanho médio do superpixel (medido em pixels). O segundo, o fator de compactação, no qual equilibra a proximidade do espaço de cores com a regularização espacial, ou seja, quanto menor esse parâmetro mais ajustado ao espaço de cores será o superpixel. A escolha de qual algoritmo de agrupamento (SLIC e SLICO), parâmetros (tamanho médio do superpixel e o fator de compactação) e o modo da imagem de RM (FLAIR, T1, T1c, T2) que serão aplicados implicam diretamente na qualidade da segmentação da lesão, pois cada sub-região da lesão pode estar melhor representada em um determinado modo de RM. Portanto, a fim de otimizar tais parâmetros, o algoritmo de otimização de enxame de partículas, do inglês Particle Swarm Optimization (PSO), foi utilizado para buscar os melhores parâmetros de forma automática.

O algoritmo PSO [Eberhart and Kennedy 1995] é uma técnica evolutiva inspirada no comportamento colaborativo de populações biológicas. O PSO pode gerar uma solução de alta qualidade dentro de um tempo de execução mais curto e exibindo uma característica de convergência estável mais eficaz do que outras técnicas de 
otimização [Silva et al. 2018]. Além disso, há menos parâmetros de controle para ajustar, e é mais eficiente em manter a diversidade do enxame, pois todas as partículas usam as informações relacionadas à partícula de maior sucesso para melhorar.

Os parâmetros a serem otimizados pelo PSO são apresentados na Tabela 1. O modo da imagem de RM pode ser do tipo FLAIR, T1, T1c e T2, a técnica de superpixel pode ser o SLIC ou o SLICO, o tamanho do superpixel pode variar de 4 a 10, e por fim, o fator de compactação pode variar de 0 a 10. Tais intervalos de valores foram definidos baseados nas propriedades das imagens de RM do cérebro. Portanto, cada partícula é definida por uma lista de quatro valores, cada uma definida pelo seu intervalo.

Tabela 1. Parâmetros otimizados pelo algoritmo PSO.
\begin{tabular}{|l|l|}
\hline Parâmetros & Valores \\
\hline Modo da RM & Flair, T1, T1c, T2 \\
\hline Técnica de superpixel & SLIC ou SLICO \\
\hline Tamanho do superpixel & 4 a 10 \\
\hline Fator de compactação & 0 a 10 \\
\hline
\end{tabular}

Após a definição dos parâmetros e seus intervalos, é definido um enxame inicial com 10 partículas, sendo 9 partículas iniciadas de maneira aleatória e 1 partícula iniciada manualmente, considerada como uma boa partícula, que foi definida empiricamente com base nos primeiros testes com o PSO, onde todas as 10 partículas eram iniciadas aleatoriamente. Para a avaliação da função objetivo foi usado o coeficiente dice entre a segmentação da lesão com tais parâmetros e a marcação dos especialistas, usando 10 pacientes, também escolhidos aleatoriamente.

Para cada uma das sub-regiões da lesão (necrose, edema, núcleo sólido e núcleo) foram otimizados um conjunto de parâmetros de forma individual. No entanto, deve-se escolher uma única partícula que melhor represente todas as sub-regiões. Para essa tarefa, foi testada cada partícula, isoladamente, em todas as outras classes. Foram usadas a média e o desvio padrão entre as classes para a escolha da melhor partícula.

\subsubsection{Classificação dos superpixels}

Logo após a otimização dos superpixels e a definição da melhor partícula, os superpixels são apresentados a uma rede geradora adversária com classificador auxiliar, do inglês Auxiliary Classifier Generative Adversarial Networks (ACGAN), para a classificação das quatro sub-regiões do tumor cerebral.

A ACGAN é uma variante da GAN [Goodfellow et al. 2014]. A diferença reside no fato que a informação sobre as classes presentes no treino podem ser adicionadas contribuindo com o processo de geração e com a estabilização da rede durante $o$ treinamento, ou seja, permitem que o modelo seja condicionado em informações para melhorar a qualidade das amostras geradas. O discriminador produz o rótulo da classe, além da decisão real ou falsa [Odena et al. 2017].

Uma vez que a ACGAN é uma técnica de aprendizado de máquina supervisionado, é necessário rotular cada um dos superpixels extraídos. Para isso, utilizou-se a marcação 
dos especialistas. A sub-região que possuir a maior área dentro do superpixel será o seu rótulo. Após esse processo, foram rotulados 8.114, 177.599, 22.443, 59.551 superpixels das sub-regiões necrose, edema, núcleo sólido, e núcleo, respectivamente.

A ACGAN utilizada neste trabalho tem duas redes neurais convolucionais com as seguintes arquiteturas. A rede discriminante é composta por cinco saídas, onde uma é responsável em discriminar se o dado recebido é verdadeiro (vindo da base de dados) ou falso (gerado pela rede geradora), as outras quatro saídas são as probabilidades do superpixel pertencer a uma das quatro sub-regiões (necrose, edema, núcleo sólido, núcleo). A rede geradora, por sua vez, é composta por uma imagem de ruído e um rótulo (da sub-região que pertencerá a amostra gerada), gerando uma saída 10x10x4 que será entrada para a rede discriminante, como ilustrado na Figura 5.

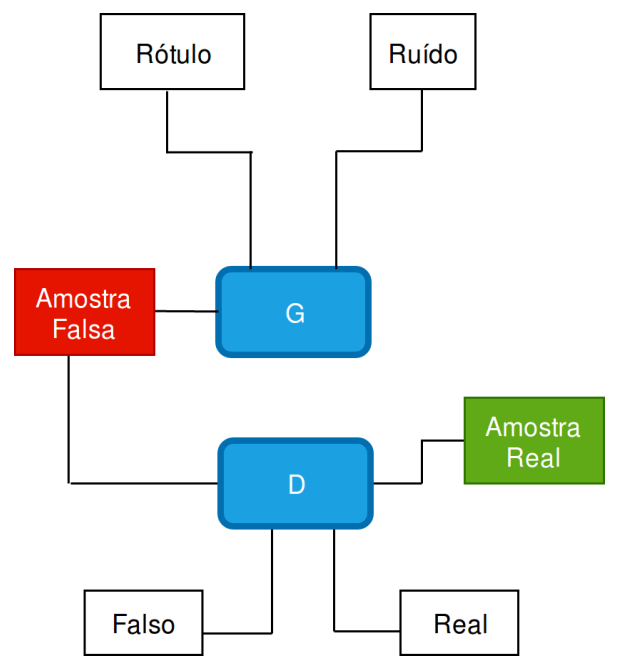

Figura 5. ACGAN e suas redes discriminante e geradora.

As Tabelas 2 e 3 apresentam as arquiteturas usadas nas duas redes presentes na ACGAN. Ambas as redes foram inspiradas na literatura, porém modificadas de forma empírica. A entrada da rede consiste no superpixel centralizado em um patch de tamanho 10x10 para todos os quatro modos de RM (FLAIR, T1, T1c e T2).

Tabela 2. Arquitetura da rede discriminante.

\begin{tabular}{|l|l|}
\hline Entrada & $10 \times 10 \times 4$ \\
\hline Convolução 1 & 64 filtros, 3x3, stride 1, Dropout + LeakyRelu \\
\hline Convolução 2 & 128 filtros, 3x3, stride 1, Dropout + LeakyRelu \\
\hline Completamente Conectada & 512 neurônios + Tangente Hiperbólica \\
\hline Saída & 5 neurônios + Softmax \\
\hline
\end{tabular}

\section{Resultados e Discussão}

Esta seção apresenta e discute os resultados obtidos com o método proposto para a segmentação de lesões no cérebro em imagens de RM 3D. A estratégia para análise dos resultados é detalhada como segue: 1) divisão da base de dados, 2) otimização da técnica de superpixel usando o algoritmo PSO, 3) classificação dos superpixels e 4) segmentação das lesões. 
Tabela 3. Arquitetura da rede geradora.

\begin{tabular}{|l|l|}
\hline Entrada & Ruído 100 \\
\hline Completamente Conectada & 1600 neurônios + BatchNormalization \\
\hline Convolução 1 & 32 filtros, 3x3, Relu + BatchNormalization + UpSampling2D \\
\hline Convolução 2 & 16 filtros, 3x3, Relu + BatchNormalization \\
\hline Convolução 3 & 4 filtros, 3x3, Tangente Hiperbólica \\
\hline Saída & 10x10x4 \\
\hline
\end{tabular}

\subsection{Divisão da base de dados}

A base de dados da BrasTS, conforme descrita na Seção 3.1, contém 220 exames de RM, juntamente com a marcação dos especialistas. Para avaliar o método proposto foram usados apenas 100 exames da base por conta da limitação de hardware. Então, a base de dados foi dividida aleatoriamente em três conjuntos: treinamento, validação e teste. $\mathrm{O}$ conjunto de treinamento contém 74 exames de RM, o conjunto de validação contém 13 exames de RM e o conjunto de testes contém 13 exames de RM.

\subsection{Otimização da técnica de superpixel usando o PSO}

A Tabela 4 apresenta os resultados do dice obtidos pelo algoritmo PSO nos exames do conjunto de 10 pacientes. Para a classe necrose a melhor partícula foi definida como T1, SLICO, 4, 4. Na classe edema a melhor partícula encontrada foi T1c, SLICO, 4, 9. Para a classe núcleo sólido a melhor partícula foi definida como T1c, SLICO, 4, 5, e por fim, na classe núcleo a melhor partícula encontrada foi T1c, SLICO, 4, 5. Após a otimização de forma individual, a partícula escolhida para as quatro sub-regiões foi T1c, SLICO, 4, 9.

Tabela 4. Resultados do algoritmo PSO na otimização dos superpixels.

\begin{tabular}{|c|c|c|c|c|c|c|}
\hline & Necrose & Edema & Núcleo sólido & Núcleo & Média & Desvio Padrão \\
\hline Necrose & 0,86 & 0,89 & 0,63 & 0,82 & 0,80 & 0,101242 \\
\hline Edema & 0,87 & 0,89 & 0,65 & 0,86 & 0,8175 & 0,097308 \\
\hline Núcleo sólido & 0,87 & 0,89 & 0,65 & 0,86 & 0,8175 & 0,097308 \\
\hline Núcleo & 0,87 & 0,89 & 0,65 & 0,86 & 0,8175 & 0,097308 \\
\hline
\end{tabular}

A finalidade da inicialização de uma partícula de forma manual para cada subregião da lesão consiste em obter uma convergência mais rápida no algoritmo PSO. O tamanho médio do superpixel nos primeiros testes foi de tamanho 7 e se observou que todas as partículas convergiam para esse tamanho. Diante disso, decidiu-se incluir essas partículas encontradas e diminuir o tamanho médio do superpixel para 4. Onde, observouse o mesmo comportamento. Quanto menor o superpixel, mais uniforme e ajustado ele será as bordas da lesão e, consequentemente, maior será o dice.

\subsection{Classificação dos superpixels}

A configuração do treinamento da ACGAN foi definido para 50 épocas com o tamanho do batch igual a 32 superpixels e a função de otimização Adam com a taxa de aprendizado de 
0,0002. Após o treinamento, a acurácia média englobando todas as sub-regiões, ou seja, todos os superpixel que foram corretamente classificados pela rede é de $87 \%$. A Tabela 5 apresenta a matriz de confusão obtida na classificação dos superpixels. A sub-região necrose obteve uma acurácia média de $67,71 \%$, a sub-região edema obteve uma acurácia média de $94,57 \%$, a sub-região núcleo sólido obteve uma acurácia média de 18,44\% e a sub-região núcleo obteve uma acurácia média de $89,35 \%$.

Tabela 5. Matriz de confusão.

\begin{tabular}{|c|c|c|c|c|}
\hline & Necrose & Edema & Núcleo Sólido & Núcleo \\
\hline Necrose & 474 & 43 & 128 & 55 \\
\hline Edema & 72 & 20782 & 325 & 797 \\
\hline Núcleo Sólido & 213 & 761 & 405 & 817 \\
\hline Núcleo & 138 & 995 & 119 & 10503 \\
\hline
\end{tabular}

Os bons resultados obtidos nas sub-regiões edema e núcleo são devidos a grande quantidade de dados presentes no treinamento da ACGAN. A classe necrose possui o menor número de instancias para o treinamento, ainda assim obteve uma acurácia maior que a região do núcleo sólido. Como visto nos resultados da matriz de confusão da Tabela 5, a classe núcleo sólido, pela sua difícil localização, confunde muitas de suas instancias com as instancias vizinhas, como a classe edema e núcleo. Exceto a classe edema que possui a maior área e é vizinha do tecido saudável, trazendo consigo informações que contribuem para sua classificação, as demais classes carecem de informações que melhor as discrimine.

\subsection{Segmentação das lesões}

A Figura 6 ilustra casos de sucesso e falha encontrados pelo método proposto. O dice médio obtido pela sub-região necrose foi de $60,35 \%$, a sub-região edema obteve um dice médio de $44,22 \%$, o dice médio obtido pela sub-região núcleo sólido foi de $16,45 \%$, e por fim, a sub-região núcleo obteve um dice médio de 31,23\%. Os baixos valores de dice demonstram a dificuldade da segmentação automática de lesões cerebrais, visto que as sub-regiões são próximas umas das outras, dificultando assim a etapa de segmentação.

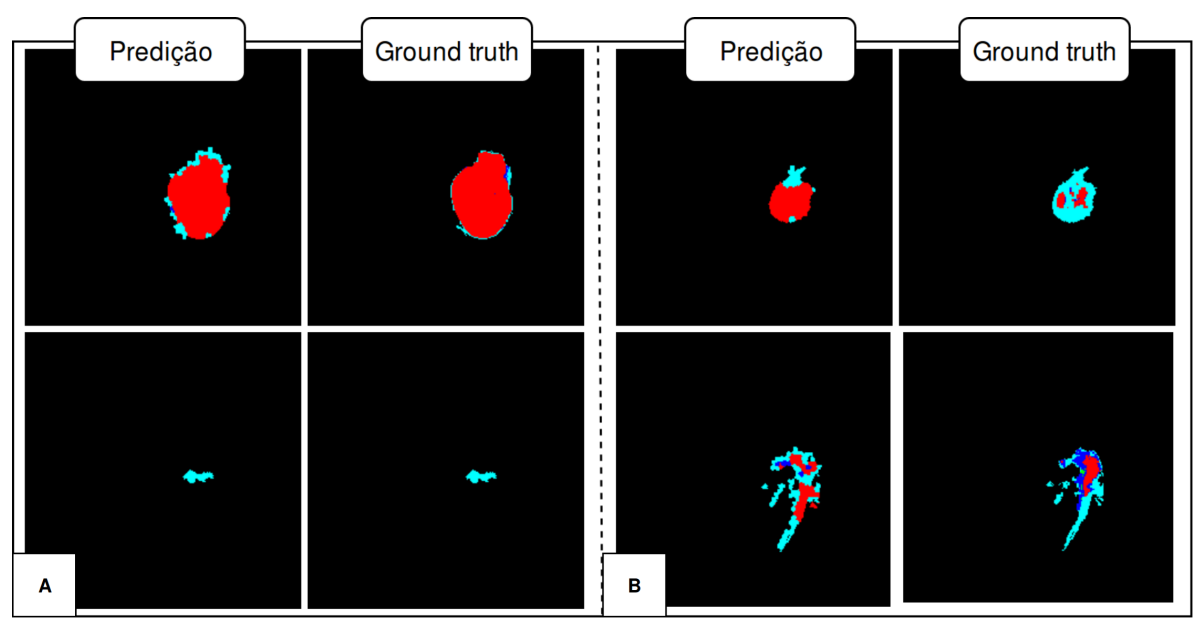

Figura 6. Casos de sucesso (A) e falha (B) na segmentação das lesões. 


\section{Conclusão}

Os tumores cerebrais são problemas graves, independente do seu diagnóstico. A detecção precoce é de extrema importância para a sobrevida do paciente. Por isso, métodos assistidos por computador são comumente desenvolvidos e a análise automática de lesões auxiliam de forma expressiva os especialistas.

Os resultados da classificação dos superpixels e a segmentação das lesões para as sub-regiões (necrose, edema, núcleo sólido e núcleo) foram promissores, obtendo uma acurácia média de $67,71 \%, 94,57 \%, 18,44 \%$ e $89,35 \%$ e dice médio de $60,35 \%, 44,22 \%$, $16,45 \%$ e $31,23 \%$, respectivamente para as quatro sub-regiões. É importante destacar que esse trabalho contou com apenas 100 exames de uma base de 220 exames disponíveis para gliomas alto grau.

Como trabalho futuro, nós identificamos alguns pontos que podem ser aprimorados a fim de obter resultados melhores. O primeiro consiste em técnicas de sobreamostragem para o balanceamento dos dados, o segundo consiste em incluir outras técnicas de superpixels na etapa de otimização, e por fim, utilizar pesos para as subregiões no treinamento da ACGAN.

\section{Referências}

Achanta, R., Shaji, A., Smith, K., Lucchi, A., Fua, P., Süsstrunk, S., et al. (2012). Slic superpixels compared to state-of-the-art superpixel methods. IEEE transactions on pattern analysis and machine intelligence, 34(11):2274-2282.

Alex, V., KP, M. S., Chennamsetty, S. S., and Krishnamurthi, G. (2017). Generative adversarial networks for brain lesion detection. In Medical Imaging 2017: Image Processing, volume 10133, page 101330G. International Society for Optics and Photonics.

Dice, L. R. (1945). Measures of the amount of ecologic association between species. Ecology, 26(3):297-302.

Diniz, P. H. B., Azevedo Valente, T. L., Bandeira Diniz, J. O., Silva, A. C., Gattass, M., Ventura, N., Muniz, B. C., and Gasparetto, E. L. (2018). Detection of white matter lesion regions in mri using slico and convolutional neural network. Computer methods and programs in biomedicine, 167:49-63.

Eberhart, R. and Kennedy, J. (1995). A new optimizer using particle swarm theory. In Micro Machine and Human Science, 1995. MHS'95., Proceedings of the Sixth International Symposium on, pages 39-43. IEEE.

Goodenberger, M. L. and Jenkins, R. B. (2012). Genetics of adult glioma. Cancer genetics, 205(12):613-621.

Goodfellow, I., Pouget-Abadie, J., Mirza, M., Xu, B., Warde-Farley, D., Ozair, S., Courville, A., and Bengio, Y. (2014). Generative adversarial nets. In Advances in neural information processing systems, pages 2672-2680.

Havaei, M., Davy, A., Warde-Farley, D., Biard, A., Courville, A., Bengio, Y., Pal, C., Jodoin, P.-M., and Larochelle, H. (2017). Brain tumor segmentation with deep neural networks. Medical image analysis, 35:18-31. 
INCA (2019). Instituto nacional do câncer, tipos de câncer: Cérebro. https://www.inca.gov.br/tipos-de-cancer/ cancer-do-sistema-nervoso-central. Accessed: 2019-01-13.

Işın, A., Direkoğlu, C., and Şah, M. (2016). Review of mri-based brain tumor image segmentation using deep learning methods. Procedia Computer Science, 102:317324.

Lefkovits, L., Lefkovits, S., and Vaida, M.-F. (2016). Brain tumor segmentation based on random forest. Memoirs of the Scientific Sections of the Romanian Academy, 39(1):8393.

Menze, B. H., Jakab, A., Bauer, S., Kalpathy-Cramer, J., Farahani, K., Kirby, J., Burren, Y., Porz, N., Slotboom, J., Wiest, R., et al. (2015). The multimodal brain tumor image segmentation benchmark (brats). IEEE transactions on medical imaging, 34(10):1993.

Morris, S. A. and Slesnick, T. C. (2018). Magnetic resonance imaging. Visual Guide to Neonatal Cardiology, pages 104-108.

Odena, A., Olah, C., and Shlens, J. (2017). Conditional image synthesis with auxiliary classifier gans. In Proceedings of the 34th International Conference on Machine Learning-Volume 70, pages 2642-2651. JMLR. org.

Omuro, A. and DeAngelis, L. M. (2013). Glioblastoma and other malignant gliomas: a clinical review. Jama, 310(17):1842-1850.

Pereira, S., Pinto, A., Alves, V., and Silva, C. A. (2016). Brain tumor segmentation using convolutional neural networks in mri images. IEEE transactions on medical imaging, 35(5):1240-1251.

Siegel, R. L., Miller, K. D., and Jemal, A. (2016). Cancer statistics, 2016. CA: a cancer journal for clinicians, 66(1):7-30.

Silva, G. L. F. d., Valente, T. L. A., Silva, A. C., de Paiva, A. C., and Gattass, M. (2018). Convolutional neural network-based pso for lung nodule false positive reduction on ct images. Computer methods and programs in biomedicine, 162:109-118.

Stewart, B., Wild, C. P., et al. (2014). World cancer report 2014. 